\title{
An Overview of Intravitreal Aflibercept in Diabetic Macular Oedema
}

\author{
Jean-François Korobelnik ${ }^{1-3}$ and Sebastian Wolf ${ }^{4}$
}

1. Professor, Université de Bordeaux, Bordeaux, France; 2. INSERM (Institut National de la Santé et de la Recherche Médicale), ISPED (Institut de Santé

Publique d'Épidémiologie et de Développement), Centre INSERM U897-Epidemiologie-Biostatistique, Bordeaux, France; 3. Centre Hospitalier Universitaire (CHU) de Bordeaux, Service d'Ophtalmologie, Bordeaux, France; 4. Director and Chief Physician, Department of Ophthalmology, Inselspital, University Hospital and University Bern, Bern, Switzerland

\begin{abstract}
Diabetic macular oedema (DMO) is responsible for much of the vision loss associated with diabetic retinopathy, which is the leading cause of blindness in working-age adults. Approximately 21 million people worldwide have DMO. The standard of care for DMO has been laser photocoagulation: this option has only a limited ability to reverse vision loss and is associated with a risk of scarring and complications. Corticosteroids have also been used in DMO treatment, but are associated with side effects such as elevated intraocular pressure and cataract. Knowledge of the role of vascular endothelial growth factor (VEGF) in the pathogenesis of DMO has led to the widespread use of anti-VEGF agents. Intravitreal bevacizumab, which is not approved for any retinal disorders, is used off-label for the treatment of DMO. Intravitreal ranibizumab (IVT-AFL) (once monthly) and intravitreal aflibercept (once every 2 months after a loading dose) have been approved for the treatment of DMO. This review summarises the clinical evidence supporting the role of IVT-AFL in the treatment of DMO. Analysis of efficacy and safety data suggests that aflibercept could be used as a first-line treatment in DMO.
\end{abstract}

\section{Keywords}

Diabetic macular oedema (DMO), diabetic retinopathy, intravitreal aflibercept, placental growth factor (PIGF) inhibitor, vascular endothelial growth factor (VEGF) inhibitor

\begin{abstract}
Disclosure: Jean-François Korobelnik is a consultant for Alcon, Alimera, Allergan, Bayer, Horus, Novartis, Roche, Thea and Zeiss. Sebastian Wolf is a consultant and/or advisory board member for Alcon, Allergan, Bayer HealthCare, Heidelberg Engineering, Novartis, Roche and Zeiss.

Open Access: This article is published under the Creative Commons Attribution Noncommercial License, which permits any non-commercial use, distribution, adaptation and reproduction provided the original author(s) and source are given appropriate credit.

Received: 26 March 2015 Accepted: 11 May 2015 Citation: European Ophthalmic Review, 2015;9(1):37-41 DOI: 10.17925/EOR.2015.09.01.37

Correspondence: Jean-François Korobelnik, Service d’Ophtalmologie Hôpital Pellegrin, CHU de Bordeaux, Place Amélie Raba Léon, 33000 Bordeaux, France.

E: jean-francois.korobelnik@chu-bordeaux.fr

Support: Editorial support was provided by Michelle Olsher of PAREXEL, which was funded by Bayer Pharma AG. No publication fees were associated with the publication of this article.
\end{abstract}

Diabetic macular oedema (DMO) is responsible for much of the vision loss associated with diabetic retinopathy, the leading cause of blindness in working-age adults. ${ }^{1-3} \mathrm{DMO}$ is characterised by thickening of the central retina due to leakage of fluid from blood vessels, and can occur at any stage of retinopathy. ${ }^{4}$ Recent estimates suggest that around 21 million people worldwide have DMO. ${ }^{5}$

Evaluation of DMO should be based on both functional (visual acuity) and anatomical criteria. Optical coherence tomography (OCT) is used to assess retinal morphology and thickness of the macula, and additional methods for anatomical assessment include biomicroscopy, fundus photography and fluorescein angiography. ${ }^{6,7}$ Although focal laser photocoagulation has been the standard of care for DMO, it can only slow progression and its ability to reverse vision loss is low; 8,9 in addition, it has been associated with a risk of scarring and other thermal complications. 10,11 The corticosteroids (dexamethasone, triamcinolone acetonide and fluocinolone acetonide) have also been used for treatment of persistent or refractory DMO and provide improvements in retinal thickness and visual acuity. However, steroidrelated side effects, such as elevated intraocular pressure and cataract, must be considered. ${ }^{12,13}$
Awareness of the role that vascular endothelial growth factor (VEGF) plays in the pathogenesis of retinal diseases, including $\mathrm{DMO}^{14-16}$ has led to the development and widespread use of VEGF inhibitors in this area. Intravitreal ranibizumab (IVR) has been approved for the treatment of DMO with once-monthly dosing, whereas intravitreal aflibercept (IVT-AFL) has been approved with dose administration once every 2 months after a loading dose. IVT-AFL has recently been approved in the EU and the US for the treatment of patients with visual impairment due to DMO. ${ }^{17,18}$ Further regulatory submissions for IVT-AFL in DMO have been made in Japan, Asia-Pacific and Latin America. In addition to IVR and IVT-AFL, intravitreal bevacizumab (IVB), which is not approved for any retinal disorders, is often used off-label for the treatment of DMO. This review summarises the evidence supporting the role of IVT-AFL in the treatment of DMO.

\section{How Does Intravitreal Aflibercept Work?}

Aflibercept is composed of extracellular domains from human VEGF receptors 1 and 2 fused to the Fc portion of human immunoglobulin-G1. ${ }^{19}$ By contrast, bevacizumab is a recombinant, humanised monoclonal antibody that binds all isoforms of VEGF-A, and ranibizumab is a humanised anti-VEGF antibody fragment comprising the antigen- 


\section{Table 1: Major Clinical Studies of Intravitreal Aflibercept for the Treatment of Diabetic Macular Oedema}

\begin{tabular}{|c|c|c|c|c|c|}
\hline Study and Reference & Patients & Design & Outcomes & Efficacy & Conclusions \\
\hline \multicolumn{6}{|l|}{ Phase II } \\
\hline DA VINCl ${ }^{25}$ & $\begin{array}{l}221 \text { patients randomised, } \\
\text { 1:1:1:1:1 to the following } \\
\text { five groups: } \\
\text { IVT-AFL } 0.5 q 4 ; \\
\text { IVT-AFL 2q4; } \\
\text { IVT-AFL 2q8; } \\
\text { IVT-AFL 2PRN; } \\
\text { macular laser } \\
\text { (study treatment applied } \\
\text { to one eye per patient) }\end{array}$ & $\begin{array}{l}\text { Multicentre, randomised, } \\
\text { double-masked clinical } \\
\text { trial; analysis of data at } \\
24 \text { weeks post-baseline }\end{array}$ & $\begin{array}{l}\text { Proportion of eyes gaining } \\
\geq 15 \text { ETDRS letters in } \\
\text { BCVA from baseline } \\
\text { Reduction from baseline } \\
\text { in CRT }\end{array}$ & $\begin{array}{l}\text { IVT-AFL: }+8.5 \text { to }+11.4 \\
\text { Laser: }+2.5 \\
\text { ( } \mathrm{p}=0.0085 \text { for each } \\
\text { IVT-AFL group versus } \\
\text { laser group) } \\
\text { IVT-AFL: } 17-34 \% \\
\text { Laser: } 21 \% \\
\text { IVT-AFL: } 127.3-194.5 \mu \mathrm{m} \\
\text { Laser: } 67.9 \mu \mathrm{m}\end{array}$ & $\begin{array}{l}\text { Compared with macular } \\
\text { laser photocoagulation, } \\
\text { IVT-AFL produced a } \\
\text { statistically significant } \\
\text { and clinically relevant } \\
\text { improvement in BCVA } \\
\text { Results suggest potential } \\
\text { to reduce treatment } \\
\text { burden by prolonging } \\
\text { retreatment interval from } \\
4 \text { to } 8 \text { weeks }\end{array}$ \\
\hline $\begin{array}{l}\text { DA VINCI } \\
(1-\text { year outcomes })^{26}\end{array}$ & $\begin{array}{l}\text { As in DA VINCI study } \\
\text { above }\end{array}$ & $\begin{array}{l}\text { Continuation of } \\
\text { DA VINCI study above; } \\
\text { analysis of data at } 52 \\
\text { weeks post-baseline }\end{array}$ & $\begin{array}{l}\text { Mean change in BCVA } \\
\text { from baseline } \\
\text { Proportion of eyes } \\
\text { gaining } \geq 15 \text { ETDRS letters } \\
\text { in BCVA from baseline } \\
\text { Reduction from baseline } \\
\text { in CRT }\end{array}$ & $\begin{array}{l}\text { IVT-AFL: }+9.7 \text { to +13.1 } \\
\text { Laser: }-1.3 \\
\text { IVT-AFL: } 23.8-45.5 \% \\
\text { Laser: } 11.4 \% \\
\text { IVT-AFL: } 165.4-227.4 \mu \mathrm{m} \\
\text { Laser: } 58.4 \mu \mathrm{m}\end{array}$ & $\begin{array}{l}\text { BCVA gains observed at } \\
24 \text { weeks were maintained } \\
\text { or improved at } 52 \text { weeks } \\
\text { in all IVT-AFL groups }\end{array}$ \\
\hline \multicolumn{6}{|l|}{ Phase III } \\
\hline $\begin{array}{l}\text { VISTA-DME and } \\
\text { VIVID-DME (1-year } \\
\text { outcomes) }^{27}\end{array}$ & $\begin{array}{l}872 \text { patients (466 in VISTA- } \\
\text { DME; } 406 \text { in VIVID-DME) } \\
\text { randomised 1:1:1 to the } \\
\text { following three groups: } \\
\text { IVT-AFL 2q4 } \\
\text { IVT-AFL 2q8 } \\
\text { macular laser } \\
\text { (study treatment } \\
\text { applied to one eye } \\
\text { per patient) }\end{array}$ & $\begin{array}{l}\text { US-based (VISTA-DME) } \\
\text { and international } \\
\text { (VIVID-DME; Europe, } \\
\text { Japan and Australia), } \\
\text { multicentre, randomised, } \\
\text { double-masked clinical } \\
\text { trials; analysis of data at } \\
52 \text { weeks post-baseline }\end{array}$ & $\begin{array}{l}\text { Proportion of eyes gaining } \\
\geq 15 \text { ETDRS letters in } \\
\text { BCVA from baseline }\end{array}$ & 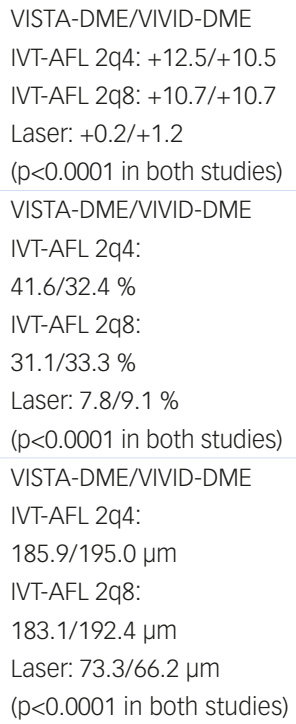 & $\begin{array}{l}\text { IVT-AFL was superior to } \\
\text { macular laser in terms } \\
\text { of both functional and } \\
\text { anatomical endpoints at } \\
\text { week } 52 \text {. Efficacy was } \\
\text { similar in the } 2 \text { q4 and } \\
2 \text { q8 groups despite the } \\
\text { latter having a prolonged } \\
\text { retreatment interval }\end{array}$ \\
\hline
\end{tabular}

$B C V A=$ best-corrected visual acuity; $C R T=$ central retinal thickness; ETDRS = Early Treatment Diabetic Retinopathy Study; IVT-AFL = intravitreal aflibercept; $2 P R N=2$ mg for three initial doses then as needed; $0.5 q 4=0.5 \mathrm{mg}$ every 4 weeks; $2 q 4=2 \mathrm{mg}$ every 4 weeks; $298=2 \mathrm{mg}$ every 8 weeks after three (DA VINCI) or five (VISTA-DME/VIVID-DME) initial monthly doses.

binding Fab moiety, without the Fc domain, which was derived from bevacizumab. ${ }^{20,21}$ These differences in design result in substantial differences in the pharmacokinetic properties of these drugs.

Aflibercept binds human VEGF-A with a markedly (approximately 100fold) higher affinity than ranibizumab or bevacizumab: an in vitro binding study using the VEGF- $\mathrm{A}_{165}$ isoform reported a dissociation constant $\left(\mathrm{K}_{\mathrm{D}}\right)$ of $0.49 \mathrm{pM}$ for aflibercept compared with $46 \mathrm{pM}$ for ranibizumab and $58 \mathrm{pM}$ for bevacizumab. ${ }^{21}$ In addition, VEGF receptor 1 and 2 activation and VEGF-A-induced calcium mobilisation and migration in human umbilical vein endothelial cells (HUVECS) were more potently inhibited by aflibercept than by ranibizumab or bevacizumab. ${ }^{2}$

Of particular relevance is the synergistic relationship between VEGF and placental growth factor (PIGF) in the development of angiogenesis, including the disruption of the retinal barrier during diabetic retinopathy. ${ }^{16,22}$ In vitro studies showed that aflibercept binds PIGF-2 $\left(K_{D}: 38.9 \mathrm{pM}\right)$, whereas ranibizumab and bevacizumab do not. Consistent with this finding, only aflibercept inhibited PIGF-induced VEGF receptor 1 activation and HUVEC migration. ${ }^{21}$ In immortalised bovine retinal endothelial cells, proliferation induced by a combination of VEGF-A and PIGF was completely suppressed by aflibercept, ${ }^{23}$ but only partially suppressed by ranibizumab. ${ }^{24} \mathrm{All}$ three compounds have been shown to reduce VEGF-A-stimulated migration, ${ }^{23,24}$ and therapeutically achievable concentrations of aflibercept not only normalised VEGF-A-stimulated cell migration but drove it below the level of basal migration. ${ }^{23}$

\section{Clinical Benefits of Intravitreal Aflibercept}

The benefits of IVT-AFL in DMO have been established in three major studies: the phase II DMO and VEGF Trap-Eye: Investigation of Clinical 
Impact (DA VINCI) study 25,26 and the phase III studies Study of Intravitreal Administration of VEGF Trap-Eye [BAY86-5321] in Patients With Diabetic Macular Edema (VISTA-DME) and Intravitreal Aflibercept Injection in Vision Impairment Due to Diabetic Macular Edema (VIVID-DME; see Table 1). ${ }^{27}$ VISTA-DME and VIVID-DME had similar study designs but enrolled patients from different regions. All three studies enrolled adult patients with type 1 or type 2 diabetes, DMO involving the central macula and a best-corrected visual acuity (BCVA) of 73-24 letters on the Early Treatment Diabetic Retinopathy Study (ETDRS) scale (approximate Snellen equivalent: 20/40-20/320). The studies were active-controlled, comparing IVT-AFL with macular laser photocoagulation. Four IVT-AFL regimens were tested in the DA VINCI study $(0.5 \mathrm{mg}$ every 4 weeks; 2 mg every 4 weeks [2q4]; 2 mg every 8 weeks [2q8] after three initial monthly doses; 2 mg as needed after three initial monthly doses), and two regimens were tested in the VISTA-DME/VIVID-DME studies (2q4; 2q8 after five initial monthly doses).

\section{Efficacy}

In the DA VINCI study, patients receiving IVT-AFL experienced significantly greater ETDRS letter gains from baseline than patients receiving macular laser photocoagulation (see Table 1); these gains were observed at 24 weeks post-baseline with each IVT-AFL regimen tested, ${ }_{1}^{25}$ and were maintained or improved at 52 weeks post-baseline. ${ }^{26}$ Improvements in vision were accompanied by significant reductions in central retinal thickness (CRT; see Table 1), and at week 52, the Diabetic Retinopathy Severity Score (DRSS) had improved from baseline in 31-64\% of patients receiving IVT-AFL compared with only $12 \%$ of patients receiving laser treatment.

The phase III studies VISTA-DME and VIVID-DME have confirmed the promising findings of the DA VINCI study. At 52 weeks, IVT-AFL was superior to laser photocoagulation in improving BCVA and CRT from baseline (see Table 1 and Figure 1). Compared with the respective laser treatment groups, a significantly greater proportion of patients in the IVT-AFL groups experienced improvements from baseline in BCVA of 15 ETDRS letters or more in the VISTA-DME and VIVID-DME studies (see Table 1). The proportion of patients showing an improvement in the DRSS was also significantly greater in the IVT-AFL groups than in the laser groups: in VISTA-DME, an improvement of two steps or more was observed in $33.8 \%$ and $29.1 \%$ of the IVT-AFL $2 \mathrm{q} 4$ and $2 \mathrm{q} 8$ groups, respectively, compared with $14.3 \%$ of the laser group $(p<0.01)$; the corresponding proportions in VIVID-DME were $33.3 \%$ and $27.7 \%$ in the IVT-AFL groups compared with $7.5 \%$ in the laser group $(p<0.001) .{ }^{27}$ Although not yet fully published, data on longer-term outcomes in the VISTA-DME and VIVID-DME trials suggest that the statistically significant benefits over laser achieved at week 52 were maintained through 100 weeks. $^{28}$

IVT-AFL has been indirectly compared with IVR in several meta-analyses A cochrane meta-analysis found no significant difference between IVTAFL and IVR in terms of the percentage of patients experiencing an improvement in BCVA of 15 or more ETDRS letters, although the authors noted that there was little power to detect such a difference. ${ }^{29}$ Another meta-analysis reported that IVR was numerically but not statistically superior to IVT-AFL in terms of the percentage of patients experiencing an improvement in BCVA of 10 or more ETDRS letters. ${ }^{30}$ Analysis of the mean change from baseline in BCVA has produced more consistent results, with two meta-analyses reporting a greater degree of improvement with IVT-AFL than with IVR. ${ }^{29,31}$ The most recent meta-analysis, which used a robust methodology to identify a network of up to 10 studies involving

\section{Figure 1: Mean Change from Baseline in BCVA and CRT in Phase III Trials of Aflibercept for the Treatment of Diabetic Macular Oedema (1-year Data From VISTA-DME and VIVID-DME)}
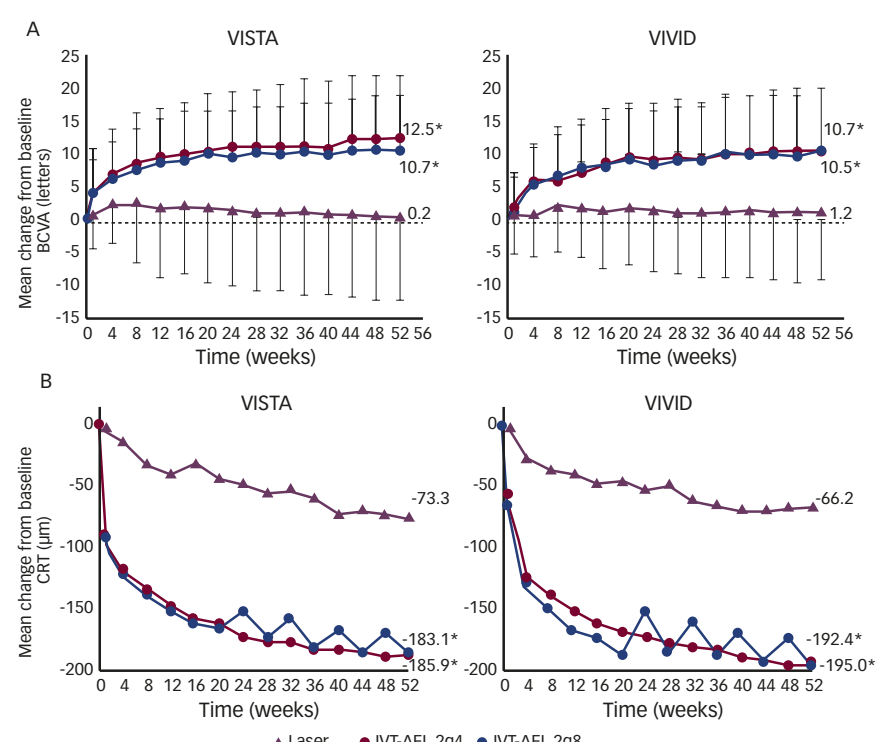

$B C V A$ = best-corrected visual acuity; $C R T=$ central retinal thickness; IVT-AFL intravitreal aflibercept injection; $2 q 4=2 \mathrm{mg}$ every 4 weeks; $2 q 8=2 \mathrm{mg}$ every 8 weeks after five initial monthly doses. ${ }^{*} p<0.0001$ versus laser. Reproduced with permission from Korobelnik et al. 2014. ${ }^{27}$

3,060 patients with DMO, showed that IVT-AFL 2 q8 improved the mean 12-month change in BCVA to a greater extent than IVR $0.5 \mathrm{mg}$ pro re nata; there was a four- to fivefold difference between the two agents in both mixed-treatment comparisons (mean difference was 4.67 letters) and in Bucher indirect comparisons (mean difference was 4.82 letters). ${ }^{31}$

IVT-AFL has also been directly compared with IVR and IVB in a recent US National Institutes of Health-sponsored trial: the Diabetic Retinopathy Clinical Research Network (DRCR.net) comparative effectiveness study in patients with DMO (Protocol T). ${ }^{32}$ In this study, 660 adults with DMO were randomised to receive IVT-AFL $2.0 \mathrm{mg}$, IVR $0.3 \mathrm{mg}$ or IVB $1.25 \mathrm{mg}$. Over 52 weeks, IVT-AFL produced a statistically significant two-letter BCVA benefit over IVR (13.3 versus 11.2 mean letter gain; $p=0.03$ ) and a statistically significant $>3$ letter BCVA benefit over IVB (13.3 versus 9.7 mean letter gain; $p<0.001)$. These BCVA gains were achieved with a median of nine IVT-AFL injections versus 10 IVR and 10 IVB injections. Moreover, a significantly smaller proportion of patients receiving IVT-AFL required laser photocoagulation (at least once between 24 and 48 weeks) versus those receiving IVR or IVB (37 \% versus $46 \%$ or $56 \%$; $\mathrm{p}<0.001$ for overall comparison).

The benefits of IVT-AFL in the Protocol T study were primarily driven by the patients with worse visual acuity at baseline. ${ }^{32}$ In the subgroup with better baseline vision (initial visual acuity letter score 78-69 or approximately 20/32-20/40), the mean improvement was 8.0 with IVT-AFL, 8.3 with IVR and 7.5 with IVB ( $p>0.50$ for each pairwise comparison). However, in the subgroup with worse baseline vision (initial letter score less than 69 or approximately 20/50 or worse), IVT-AFL produced statistically significant and clinically meaningful benefits of approximately five letters over IVR (18.9 versus 14.2 mean letter gain; $p=0.003)$ and approximately seven letters over IVB (18.9 versus 11.8 mean letter gain; $p<0.001$ ). Of note, a 15-letter gain was observed in $34 \%$ more IVT-AFL-treated eyes than IVR-treated eyes 


\section{Table 2: Overview of Safety Data from Major Clinical Studies of Intravitreal Aflibercept for the Treatment of Diabetic Macular Oedema}

\begin{tabular}{|c|c|c|c|c|c|c|}
\hline \multirow{3}{*}{$\begin{array}{l}\text { Study } \\
\text { Treatment }\end{array}$} & \multicolumn{6}{|c|}{ Patients with Adverse Event, n (\%) } \\
\hline & \multicolumn{3}{|c|}{ DA VINCI (1-year Outcomes) ${ }^{26}$} & \multicolumn{3}{|c|}{ VISTA-DME + VIVID-DME (1-year Outcomes) ${ }^{27}$} \\
\hline & Laser $(n=44)$ & IVT-AFL all $(\mathrm{n}=175)$ & IVT-AFL 2q8 (n=42) & Laser $(n=287)$ & IVT-AFL 2q4 (n=291) & IVT-AFL 2q8 $(n=287)$ \\
\hline \multicolumn{7}{|l|}{ Common ocular adverse events* } \\
\hline Conjunctival haemorrhage & $8(18.2 \%)$ & 47 (26.9 \%) & 15 (35.7 \%) & 50 (17.4 \%) & 89 (30.6 \%) & $73(25.4 \%)$ \\
\hline Eye pain & $2(4.5 \%)$ & $24(13.7 \%)$ & $6(14.2 \%)$ & $18(6.3 \%)$ & $31(10.7 \%)$ & $21(7.3 \%)$ \\
\hline Vitreous floaters & $2(4.5 \%)$ & $12(6.9 \%)$ & $2(4.8 \%)$ & $9(3.1 \%)$ & $21(7.2 \%)$ & $13(4.5 \%)$ \\
\hline Intraocular pressure increased & $1(2.3 \%)$ & $17(9.7 \%)$ & $4(9.5 \%)$ & $10(3.5 \%)$ & $17(5.8 \%)$ & $12(4.2 \%)$ \\
\hline Cataract & $2(4.5 \%)$ & $12(6.9 \%)$ & $2(4.8 \%)$ & $15(5.2 \%)$ & $15(5.2 \%)$ & $14(4.9 \%)$ \\
\hline \multicolumn{7}{|c|}{ Additional adverse events of interest } \\
\hline Endophthalmitis & 0 & $2(1.1 \%)$ & 0 & 0 & 0 & 0 \\
\hline $\begin{array}{l}\text { Arterial thromboembolic events } \\
\text { (APTC definition) }\end{array}$ & - & - & - & $8(2.8 \%)$ & $9(3.1 \%)$ & $10(3.5 \%)$ \\
\hline Non-fatal myocardial infarction & - & - & - & $5(1.7 \%)$ & $4(1.4 \%)$ & $3(1.0 \%)$ \\
\hline Non-fatal stroke & - & - & - & $2(0.7 \%)$ & $3(1.0 \%)$ & $5(1.7 \%)$ \\
\hline Vascular death & - & - & - & $2(0.7 \%)$ & $2(0.7 \%)$ & $2(0.7 \%)$ \\
\hline
\end{tabular}

*Ocular adverse events occurring in at least $5 \%$ of patients receiving intravitreal aflibercept (IVT-AFL) in VISTA-DMENVIVID-DME. APTC = Anti-Platelet Trialists' Collaboration; 2 $4=$ 2 mg every 4 weeks; $298=2$ mg every 8 weeks after three (DA VINCI) or five (VISTA-DME/VIVID-DME) initial monthly doses.

(67\% versus $50 \%$ ) and $63 \%$ more IVT-AFL-treated eyes than IVBtreated eyes (67\% versus $41 \%)$. In addition, among the subgroup with worse baseline vision, visual acuity benefits of IVT-AFL over IVR and IVB were demonstrated as early as 4 weeks after treatment initiation. Improvements in terms of decreased CRT mirrored those observed for visual acuity outcomes, with IVT-AFL showing greatest benefits over IVR and IVB among the subgroup with worse baseline vision..$^{22}$ When considering the results of the Protocol T study, it should be noted that although the DRCR.net trial used the $0.3 \mathrm{mg}$ dose of IVR (not the $0.5 \mathrm{mg}$ dose that is approved in Europe), previous evidence suggests that there is no meaningful difference in efficacy outcomes between the two IVR doses. ${ }^{33}$

\section{Safety}

An overview of 1-year safety data from the DA VINCI, VISTA-DME and VIVID-DME studies is presented in Table 2. The overall incidence of ocular adverse events (AES) in the VISTA-DME and VIVID-DME studies was similar across treatment groups, and no clinically relevant differences were observed in the frequency or pattern of serious ocular AEs. The incidence of endophthalmitis - a potential complication of intravitreal injections ${ }^{34}$ - was low, with only two cases reported across the study programme (a third patient in the DA VINCI study ${ }^{25,26}$ developed uveitis). Intraocular inflammation was also infrequent, occurring in $0.1-0.4 \%$ of intravitreal injections in the first 52 weeks of the VISTA-DME and VIVIDDME studies. ${ }^{27}$

Intraocular steroids have been associated with an increased risk of cataract and increased intraocular pressure..$^{12,13}$ Cataracts occurred at a similar rate in patients receiving IVT-AFL and those receiving laser treatment in the first 52 weeks of the VISTA-DME and VIVID-DME studies. ${ }^{27}$ In the DA VINCl study, increases in intraocular pressure occurred in $9.7 \%$ of patients receiving IVT-AFL compared with $2.3 \%$ of patients receiving laser treatment; however, these events occurred immediately after injection of IVT-AFL and normalised within 1 hour.. ${ }^{25}$ Although interstudy comparisons should be interpreted with caution, the rates of increased intraocular pressure in studies of IVT-AFL (see Table 2) were lower than those reported in a systematic review of randomised clinical trials of intraocular steroids (injected or implanted) for $\mathrm{DMO} .^{35}$ The review reported that increased intraocular pressure occurred in 12-65\% of treated eyes (versus 0-6\% of control groups), while 0-43\% of treated eyes had cataract progression (versus 0-14\% of controls). Increased intraocular pressure and cataract progression occurred in both steroid injection-treated and steroid implant-treated eyes. ${ }^{35}$

The possibility of systemic AEs is a concern associated with the use of intraocular anti-VEGF therapies. ${ }^{36}$ The DA VINCI study did not show any significant safety signals suggestive of an increased risk of systemic AEs in patients receiving IVT-AFL. ${ }^{25,26}$ The VISTA-DME and VIVID-DME studies also showed no clear trends between treatment groups in serious systemic AEs and arterial thromboembolic events defined by the Anti-Platelet Trialists' Collaboration (APTC; see Table 2). ${ }^{27}$ In the recent DRCR.net comparative effectiveness study in patients with DMO (Protocol T), the rates of APTC-defined arterial thromboembolic events were uniformly low: $3 \%$ in the IVT-AFL group, $5 \%$ in the IVR group and $4 \%$ in the IVB group. Overall rates of pre-specified systemic AES were similar in the three treatment groups. ${ }^{32}$ However, the ability of these clinical trials to detect rare systemic AEs may be limited by their relatively small sample sizes; ongoing surveillance will shed further light on the safety profile of IVT-AFL. ${ }^{27}$

\section{Conclusions}

IVT-AFL is a recently approved anti-VEGF treatment option for patients with visual impairment due to DMO. It has a markedly higher affinity for VEGF than IVR and IVB, and it also targets PIGF, another member of the VEGF family that has been shown to contribute to angiogenesis. In contrast to IVR (which requires once-monthly dosing), IVT-AFL has been approved with a dosing regimen of one injection every 2 months; it may therefore reduce the number of injections and office visits required, thus reducing the burden of treatment for patients, physicians and the healthcare system. 
In clinical trials, IVT-AFL improved visual acuity and CRT to a significantly greater extent than macular laser photocoagulation; it also produced improvements in the DRSS, suggesting regression of the underlying diabetic retinopathy. IVT-AFL was generally well tolerated: the rates of endophthalmitis and intraocular inflammation were low, and the rates of APTC-defined arterial thromboembolic events showed no clear trends between treatment groups. Increases in intraocular pressure in patients receiving IVT-AFL were less common than previously reported in patients receiving intraocular steroids (based on indirect study comparison) and were generally transient; the incidence of another steroid-related $A E$, cataract, showed no difference between treatment groups in the phase III trials of IVT-AFL.

Results from a recent meta-analysis and a recent direct comparative effectiveness study suggested that IVT-AFL may be superior to IVR and IVB in terms of the extent of visual and anatomical benefits. Overall, these findings highlight the role that IVT-AFL has to play in the treatment of patients with DMO
1. Boyer DS, Hopkins JJ, Sorof J, Ehrlich JS, Anti-vascular endothelial growth factor therapy for diabetic macular edema, Ther Adv Endocrinol Metab, 2013;4:151-69.

2. Centers for Disease Control and Prevention, Nationa diabetes fact sheet: National estimates and general information on diabetes and prediabetes in the United States, 2011. Atlanta: U.S. Department of Health and Human Services, Centers for Disease Control and Prevention, 2011.

3. International Diabetes Federation, IDF Diabetes Atlas, Brussels: International Diabetes Federation, 2013.

4. Fong DS, Aiello L, Gardner TW, et al., Diabetic retinopathy, Diabetes Care, 2003:26:226-9.

5. Yau JW, Rogers SL, Kawasaki R, et al., Global prevalence and major risk factors of diabetic retinopathy, Diabetes Care 2012;35:556-64

6. Midena E, Vujosevic S, Diagnosing and monitoring diabetic macular edema: structural and functional tests, int Ophthalmol, 2012 [Epub ahead of print].

7. Ghanchi F, Diabetic Retinopathy Guidelines Working Group, The Royal college of Ophthalmologists' clinical guidelines for diabetic retinopathy: a summary, Eye, 2013;27:285-7.

8. Photocoagulation for diabetic macular edema. Early Treatment Diabetic Retinopathy Study report number 1. Early Treatment Diabetic Retinopathy Study research group Arch Ophthalmol, 1985;103:1796-806

9. Cheung N, Mitchell P, Wong TY, Diabetic retinopathy, Lancet, 2010:376:124-36.

10. Kulkarni AD, Ip MS, Diabetic macular edema: therapeutic options, Diabetes Ther, 2012;3:1-14.

11. Park YG, Kim EY, Roh YJ, Laser-based strategies to treat diabetic macular edema: history and new promisin therapies, J Ophthalmol, 2014;2014:769213.

12. Al Dhibi HA, Arevalo JF, Clinical trials on corticosteroids for diabetic macular edema, World J Diabetes, 2013;4:295-302

13. Ciulla TA, Harris A, McIntyre N, Jonescu-Cuypers C, Treatment of diabetic macular edema with sustainedrelease glucocorticoids: intravitreal triamcinolone acetonide, dexamethasone implant, and fluocinolone acetonide implant Expert Opin Pharmacother, 2014;15:953-9.
14. Semenza GL, Vascular responses to hypoxia and ischemia Arterioscler Thromb Vasc Biol, 2010;30:648-52.

15. Simo R, Sundstrom JM, Antonetti DA, Ocular Anti-VEGF therap for diabetic retinopathy: the role of VEGF in the pathogenesis of diabetic retinopathy, Diabetes Care, 2014;37:893-9.

16. Miyamoto N, de Kozak Y, Jeanny JC, et al., Placental growth factor-1 and epithelial haemato-retinal barrier breakdown: potential implication in the pathogenesis of diabetic retinopathy, Diabetologia, 2007:50:461-70.

17. EYLEA summary of product characteristics, Berlin: Bayer Pharma AG, 2014.

18. EYLEA prescribing information, Regeneron Pharmaceuticals Inc. Tarrytown, NY, 2014.

19. Holash J, Davis S, Papadopoulos N, et al., VEGF-Trap: a VEGF blocker with potent antitumor effects, Proc Nat cad Sci U S A, 2002;99:11393-8

20. Magdelaine-Beuzelin C, Pinault C, Paintaud G, Watier $H$, Therapeutic antibodies in ophthalmology: old is new again mAbs, 2010;2:176-80.

21. Papadopoulos N, Martin J, Ruan Q, et al., Binding and neutralization of vascular endothelial growth factor (VEGF) and related ligands by VEGF Trap, ranibizumab and bevacizumab, Angiogenesis, 2012:15:171-85.

22. Carmeliet P, Moons L, Luttun A et al., Synergism between vascular endothelial growth factor and placental growth vascular entiothela grown factor and placental growth in pathological conditions, Nat Med, 2011;7:575-83.

23. Deissler HL, Lang GK, Lang GE, Capacity of aflibercept to counteract VEGF-stimulated abnormal behavior of retinal counteract VEGF-Stimulated abnormal behavior of retinal
microvascular endothelial cells, Exp Eye Res, 2014;122:20-31.

24. Deissler HL, Deissler H, Lang GK, Lang GE, Ranibizumab efficiently blocks migration but not proliferation induced by growth factor combinations including VEGF in retinal endothelial cells, Graefes Arch Clin Exp Ophthalmol, 2013;251:2345-53.

25. Do DV, Schmidt-Erfurth U, Gonzalez VH, et al., The DA VINCI study: phase 2 primary results of VEGF Trap-Eye in patients with diabetic macular edema, Ophthalmology, 2011;118:1819-26.
26. Do DV, Nguyen QD, Boyer D, et al., One-year outcomes of the DA VINCI study of VEGF Trap-Eye in eyes with diabetic macular edema, Ophthalmology, 2012;119:1658-65.

27. Korobelnik JF, Do DV, Schmidt-Erfurth U, et al., Intravitreal aflibercept for diabetic macular edema, Ophthalmology, 2014; 121:2247-54

28. Midena E, Korobelnik J-F. Intravitreal aflibercept for diabetic macular edema (DME): 100-week results from the VIVID-DME and VISTA-DME studies. Presented at the 14th EURETINA Congress, London, 11-14 September 2014.

29. Virgili G, Parravano M, Menchini F, Evans JR, Anti-vascular endothelial growth factor for diabetic macular oedema Cochrane Database Syst Revs, 2014;10:CD007419.

30. Regnier S, Malcolm W, Allen F, et al., Efficacy of anti-VEGF and laser photocoagulation in the treatment of visual impairment due to diabetic macular edema: a systematic review and network meta-analysis, PloS One, 2014;9:e102309.

31. Kleijnen J, Korobelnik JF, Lang SH, et al., Systematic review, meta-analysis and mixed treatment comparison of intravitreal aflibercept with other therapies in patients with diabetic macular oedema, Diabet Med, 2015;32 (Suppl. S1):190.

32. The Diabetic Retinopathy Clinical Research Network, Aflibercept, bevacizumab, or ranibizumab for diabetic macular edema, N Eng/ J Med, 2015:372:1193-1203.

33. Brown DM, Nguyen QD, Marcus DM, et al., Long-term outcomes of ranibizumab therapy for diabetic macular edema: the 36-month results from two phase III trials: RISE and RIDE, Ophthalmology, 2013;120:2013-22.

34. Shikari H, Silva PS, Sun JK, Complications of intravitrea injections in patients with diabetes, Semin Ophthalmol, 2014;29:276-89.

35. Grover D, Li TJ, Chong CC, Intravitreal steroids for macular edema in diabetes, Cochrane Database Syst Revs, 2008:CD005656

36. Csaky K, Do DV, Safety implications of vascular endothelial growth factor blockade for subjects receiving intravitreal anti-vascular endothelial growth factor therapies, Am J Ophthalmol, 2009;148:647-56. 